\title{
Search for millicharged particles in proton-proton collisions at $\sqrt{s}=13 \mathrm{TeV}$
}

\author{
A. Ball, ${ }^{1}$ G. Beauregard, ${ }^{2}$ J. Brooke, ${ }^{3}$ C. Campagnari, ${ }^{4}$ M. Carrigan, ${ }^{5}$ M. Citron, ${ }^{4}$ J. De La Haye, ${ }^{1}$ A. De Roeck, ${ }^{1}$ \\ Y. Elskens, ${ }^{6}$ R. Escobar Franco, ${ }^{4}$ M. Ezeldine, ${ }^{7}$ B. Francis, ${ }^{5}$ M. Gastal, ${ }^{1}$ M. Ghimire, ${ }^{2}$ J. Goldstein, ${ }^{3}$ F. Golf, ${ }^{8}$ J. Guiang, ${ }^{4, *}$ \\ A. Haas, ${ }^{2}$ R. Heller, ${ }^{4, \dagger}$ C. S. Hillø,${ }^{5}$ L. Lavezzo, ${ }^{5}$ R. Loos, ${ }^{1}$ S. Lowette, ${ }^{6}$ G. Magill, ${ }^{9,10}$ B. Manley, ${ }^{5}$ B. Marsh, ${ }^{4}$ \\ D. W. Miller, ${ }^{11}$ B. Odegard, ${ }^{4}$ F. R. Saab,${ }^{7}$ J. Sahili, ${ }^{7}$ R. Schmitz, ${ }^{4}$ F. Setti, ${ }^{4}$ H. Shakeshaft, ${ }^{1}$ D. Stuart, ${ }^{4}$ M. Swiatlowski, ${ }^{11,}$ \\ J. Yoo, ${ }^{4, \S}$ H. Zaraket, ${ }^{7}$ and H. Zheng ${ }^{11}$ \\ ${ }^{1}$ CERN, Geneva 1211, Switzerland \\ ${ }^{2}$ New York University, New York, New York 10012, USA \\ ${ }^{3}$ University of Bristol, Bristol BS8 1TH, United Kingdom \\ ${ }^{4}$ University of California, Santa Barbara, California 93106, USA \\ ${ }^{5}$ The Ohio State University, Columbus, Ohio 43218, USA \\ ${ }^{6}$ Vrije Universiteit Brussel, Brussel 1050, Belgium \\ ${ }^{7}$ Lebanese University, Hadeth-Beirut, Lebanon \\ ${ }^{8}$ University of Nebraska, Lincoln, Nebraska 68588, USA \\ ${ }^{9}$ McMaster University, Hamilton L8S 4L8, Canada \\ ${ }^{10}$ Perimeter Institute for Theoretical Physics, Waterloo N2L 2Y5, Canada \\ ${ }^{11}$ University of Chicago, Chicago, Illinois 60637, USA
}

(Received 13 May 2020; accepted 17 July 2020; published 6 August 2020)

\begin{abstract}
We report on a search for elementary particles with charges much smaller than the electron charge using a data sample of proton-proton collisions provided by the CERN Large Hadron Collider in 2018, corresponding to an integrated luminosity of $37.5 \mathrm{fb}^{-1}$ at a center-of-mass energy of $13 \mathrm{TeV}$. A prototype scintillator-based detector is deployed to conduct the first search at a hadron collider sensitive to particles with charges $\leq 0.1 e$. The existence of new particles with masses between 20 and $4700 \mathrm{MeV}$ is excluded at $95 \%$ confidence level for charges between $0.006 e$ and $0.3 e$, depending on their mass. New sensitivity is achieved for masses larger than $700 \mathrm{MeV}$.
\end{abstract}

DOI: 10.1103/PhysRevD.102.032002

\section{INTRODUCTION}

Over a quarter of the mass energy of the Universe is widely thought to be some kind of nonluminous "dark" matter (DM), however, all experiments to date have failed to confirm its existence as a particle, much less its properties. The possibility that DM is not a single particle, but rather a diverse set of particles with as complex a structure in their sector as normal matter, has grown in prominence in the past decade, beginning with attempts to explain observations in high-energy astrophysics experiments [1,2].

\footnotetext{
*Present address: University of California, San Diego, California 92093, USA.

${ }^{\dagger}$ Present address: Fermi National Accelerator Laboratory, Batavia, Illinois 60510, USA.

*Present address: TRIUMF, Vancouver V6T 2A3, Canada.

${ }^{\S}$ Present address: Korea University, Seoul 02841, South Korea.

Published by the American Physical Society under the terms of the Creative Commons Attribution 4.0 International license. Further distribution of this work must maintain attribution to the author(s) and the published article's title, journal citation, and DOI. Funded by SCOAP ${ }^{3}$.
}

Many experimental efforts have been launched to look for signs of a dark sector, including searches at highenergy colliders, explorations at low-energy colliders, precision tests, and effects in DM direct detection experiments (for recent reviews see Refs. [3-5]). Most of these experiments target the dark sector via a massive dark photon, in what we refer to as the "Okun phase" $[6,7]$. An alternative assumption, which we call the "Holdom phase" [7,8], results in massless dark photons. In these models the principal physical effect is that new dark sector particles that couple to the dark photon will have a small effective electric charge. These are generically called millicharged particles since a natural value for their electric charge of $Q \sim \alpha e / \pi$ arises from oneloop effects [9]. In this paper we use the symbol $\chi$ to denote a millicharged particle. For a given mass and charge, the pair production of millicharged particles at the CERN Large Hadron Collider (LHC) is almost model independent. Every standard model (SM) process that results in dilepton pairs through a virtual photon would, if kinematically allowed, also produce $\chi^{+} \chi^{-}$pairs with a cross section reduced by a factor of $(Q / e)^{2}$ and by mass-dependent factors that are well understood. 

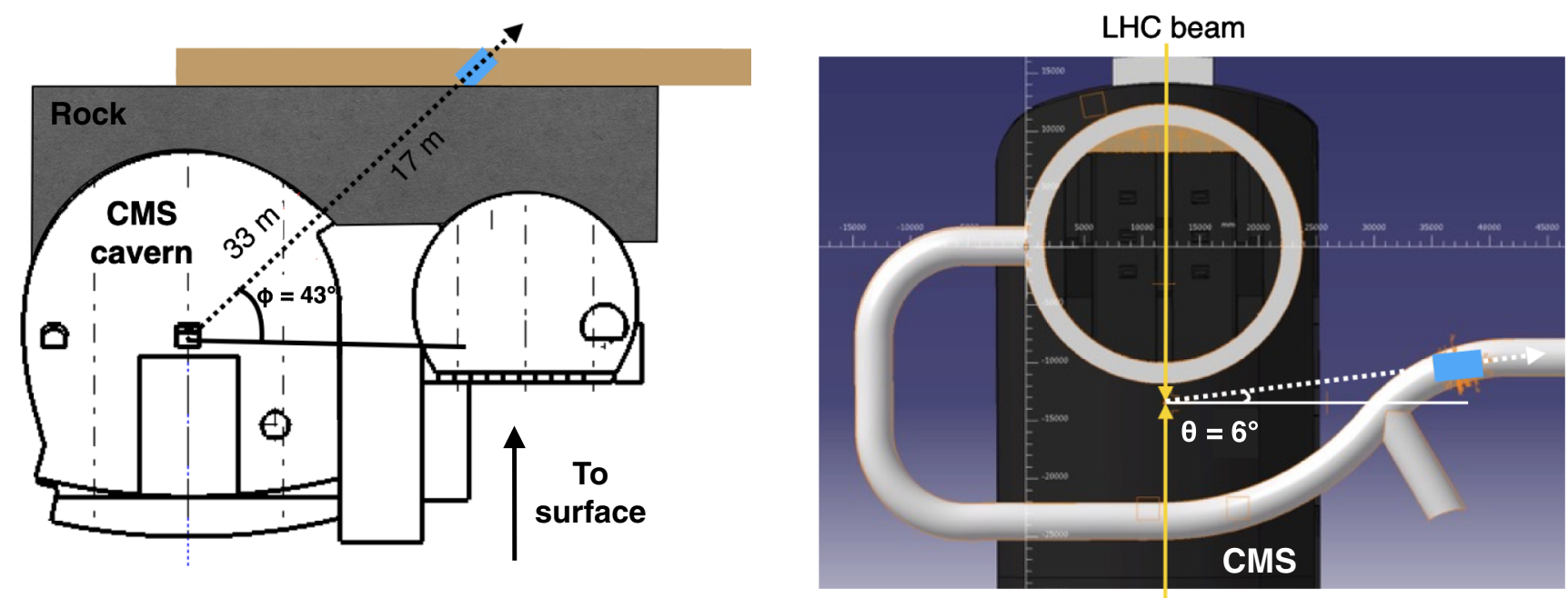

LHC beam

FIG. 1. The position of the detector, shown as a blue rectangular volume, in an elevation view (left) and plan view (right). The dashed lines represent the projection of the center of the detector to the CMS IP.

Millicharged particles can also be produced through $\mathrm{Z}$ boson couplings that depend on their hypercharge [7].

Previous experiments have searched for millicharged particles [10-17]. The parameter space spanned by the mass and charge of $\chi$ is also constrained by indirect observations from astrophysical systems [9,15,18-24], terrestrial matter [25], the cosmic microwave background (CMB) [26], big-bang nucleosynthesis [27], and universe overclosure bounds [18]. While direct searches robustly constrain the parameter space of millicharged particles, indirect observations can be evaded by adding extra degrees of freedom, which can readily occur in minimally extended dark sector models [7]. In particular, the parameter space $1<m_{\chi}<100 \mathrm{GeV}$, an ideal mass range for production at the LHC, is largely unexplored by direct (or indirect) searches. Such a signature would not be detectable by the CMS and ATLAS experiments at the LHC [28,29], as all detector elements rely on the electromagnetic (EM) interaction of the millicharged particle with ordinary matter. For a millicharged particle the interaction strength is reduced by a factor of $(Q / e)^{2}$ with respect to that of a particle of the same mass that has charge $e$. The detector signal is also reduced by the same factor, and is typically too small to be recorded by detectors designed for particles of charge $e$. The production of millicharged particles in collider experiments would result in events with missing transverse momentum, however, SM processes with neutrinos as well as instrumental effects tend to overwhelm their signatures. No searches for particles with $Q \lesssim 0.1 e$ have been performed at hadron colliders.

It is then clear that dedicated detectors are needed to search for millicharged particles at a hadron collider. In 2016, we discussed the possibility to build such a detector at the LHC, which we called milliQan [30], at the CMS experimental site and aligned with the CMS interaction point (IP). Since then we have installed and operated a small fraction of such a detector ("milliQan demonstrator") to measure backgrounds and provide a proof of principle and feedback for the full detector design. In 2018, the demonstrator collected a dataset of proton-proton ( $\mathrm{pp}$ ) interactions corresponding to an integrated luminosity of $37.5 \mathrm{fb}^{-1}$, at a center-of-mass energy of $13 \mathrm{TeV}$. This corresponds to $86 \%$ of the total luminosity delivered by the LHC in the period the demonstrator was operational. While the demonstrator is only $\sim 1 \%$ of the full milliQan, the data collected already provides competitive constraints on the existence of millicharged particles of mass $20-4700 \mathrm{MeV} / c^{2}$ and $Q / e \sim 0.01-0.3$.

\section{DETECTOR}

A thick sensitive volume is required to be capable of observing the small energy deposition of a particle with $Q \lesssim 0.1 e$. The milliQan demonstrator is, therefore, composed of three layers of $80 \times 5 \times 5 \mathrm{~cm}$ scintillator bar arrays pointing to the CMS IP, with each array consisting of three pairs of bars, stacked on a $3.6 \mathrm{~m}$ long rectangular aluminum tube, for a total of 18 bars. We label the closest, middle and furthest layer from the CMS IP as layer 1, layer 2 and layer 3, respectively.

The milliQan demonstrator is located in an underground tunnel at a distance of $33 \mathrm{~m}$ from the CMS IP, with $17 \mathrm{~m}$ of rock between the CMS IP and the demonstrator that provides shielding from most particles produced in LHC collisions. In the CMS coordinate system [29], the detector is positioned at an azimuthal angle $(\phi)$ of $43^{\circ}$ and pseudorapidity $(\eta)$ of 0.1 . Diagrams of the detector's position are shown in Fig. 1. Located $70 \mathrm{~m}$ underground, the muon flux from cosmic rays is reduced by a factor of $\sim 100$ compared to the surface. The detector is aligned 


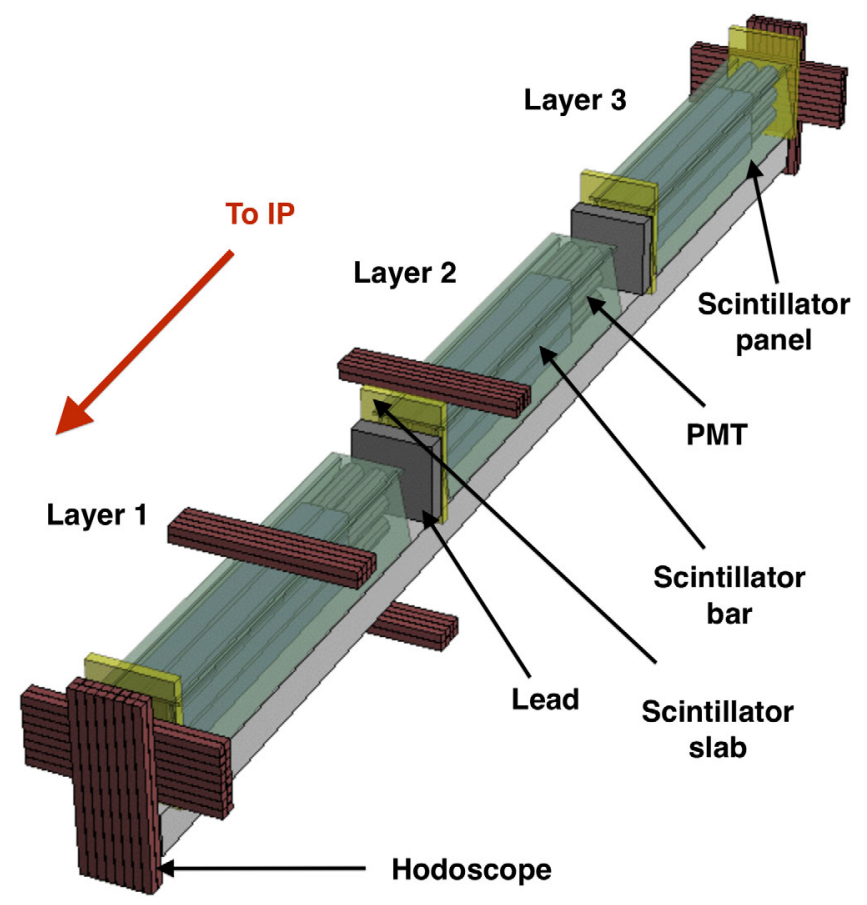

FIG. 2. A diagram of the detector components. The PMTs are not shown for the slabs or panels. All components are installed on an aluminum tube.

using standard laser-based survey techniques such that the center of the scintillator array projects a line to within $1 \mathrm{~cm}$ of the CMS IP. This alignment is validated using muons produced at the CMS IP, as discussed in Sec. IV C.

In addition to the scintillator bars, additional components were installed to reduce or characterize certain types of backgrounds. Lead bricks are placed between the layers to prevent low-energy secondary particles from one layer from entering another layer. Four scintillator slabs are located along the length of the detector to tag throughgoing particles, provide time information, and shield the bars from neutron radiation. Thin scintillator panels cover the top and sides, providing the ability to reject cosmic muons. Lastly, hodoscopes consisting of $2 \times 2 \times 45 \mathrm{~cm}$ scintillator volumes are used to identify the tracks of beam and cosmic muons. A diagram of the detector components is shown in Fig. 2, and a photograph of the installed detector is shown in Fig. 3. All scintillator volumes are comprised of Eljen EJ-200 [31].

Scintillator light in the bars, panels, and slabs is detected by photomultiplier tubes (PMTs) coupled to the scintillator volumes. Three types of PMTs are used in order to test different manufacturers and gains: the Hamamatsu R878, Hamamatsu R7725 [32], and Electron Tube 9814B [33]. Analog signals from each PMT are sent to two 16-channel CAEN V1743 digitizers [34], operating at $1.6 \times 10^{9}$ samples per second with 12-bit resolution, providing 1024 samples within a 640 ns acquisition window. Each scintillator volume with a PMT attached is referred to as a

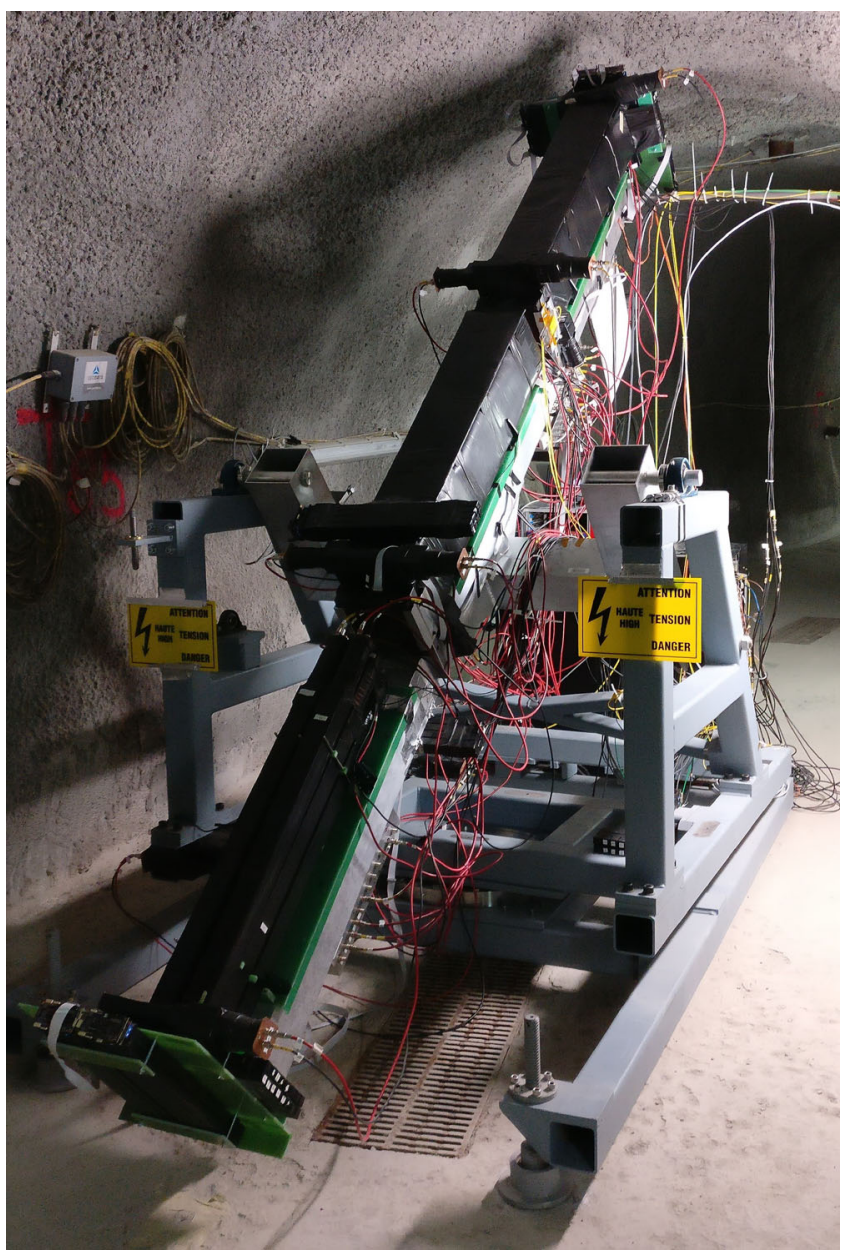

FIG. 3. A photograph of the detector, with the panels removed from layer 1 so that the bars may be seen.

"channel." Since millicharged particles produced at the CMS IP would traverse the full length of the detector in $\mathcal{O}(10) \mathrm{ns}$, tight timing requirements allow for a significant reduction in combinatoric backgrounds by requiring the coincidence of in time signals in all layers, as described in Sec. V. The PMTs are powered by a CAEN SY5527 power supply system [35]. The hodoscopes are readout with silicon photomultipliers. Their data are triggered and stored independently from the main data stream.

The dataset analyzed for this search was collected during 2018, including periods with the LHC beam providing pp collisions (the "beam-on" dataset) and periods with no collisions (the "beam-off" dataset). The beam-off dataset provides a statistically independent sample to study background processes. The detector is located in an area in the far fringe field of the CMS superconducting magnet, and both beam-on and beam-off datasets were recorded with the magnet at its nominal strength of $3.8 \mathrm{~T}$. The actual magnetic field in the milliQan cavern is measured by magnetic field sensors installed in various positions around the detector. We find this magnetic field to be under $2 \mathrm{mT}$ when the CMS 
magnet is at 3.8 T. Additional data samples were recorded with the field at $0 \mathrm{~T}$ for PMT calibration.

Data is collected by triggering on the coincidence of at least three channels (triple coincidence) within a window of 100 ns. A bar contributes to this coincidence if it has a rising edge consistent with a single photoelectron threshold, and the panels and slabs contribute to the coincidence with more stringent thresholds appropriate for identification of muons from cosmic rays ("cosmic muons") and muons from pp interactions ("beam muons"). The total trigger live times were 1106 and 1042 hours for the beam-on and beam-off datasets, respectively. The average trigger rate was $14.4 \mathrm{~Hz}$. Outside of the beam time, additional specialized runs were taken using single-channel and doublecoincidence triggers, with different thresholds and operating voltage settings, in order to collect data samples for calibrations and validation studies.

\section{CALIBRATION}

We first calibrate the size of the generated pulses in each channel, which requires a measurement of the average size of a pulse from a single photoelectron (SPE) in each PMT, as well as the mean number of photoelectrons $\left\langle\mathrm{N}_{\mathrm{PE}}\right\rangle$ generated in each channel by a throughgoing muon. The former is strictly a property of each PMT, while the latter depends on each scintillator, its wrapping, its coupling to the PMT, and the PMT quantum efficiency.

The SPE calibration is performed in situ by isolating pulses from late-arriving scintillation photons, which largely produce SPEs in the PMTs. The mean SPE area is then found by locating the peak of the resulting pulse area distribution. These measurements are cross-checked by bench measurements of SPE waveforms generated by a flashing LED, following the method outlined in Ref. [36]. In the following, the $n_{\mathrm{pe}}$ of a given pulse is defined as the pulse area divided by this per-channel SPE calibration, and represents an estimate of the true number of photoelectrons that generated the pulse.

For panels and slabs, the $\left\langle\mathrm{N}_{\mathrm{PE}}\right\rangle$ calibration is performed directly based on throughgoing beam muons (slabs) and cosmic muons (panels). The measured average pulse area is scaled by the per-channel SPE measurement to calculate the mean number of photoelectrons generated by a beam or cosmic muon.

For the bars, we use cosmic ray muons for calibration. Direct calibration with radioactive sources was not possible because the detector could not be accessed during LHC running. Since cosmic ray muons saturate the readout, we take an indirect approach, using the fact that the PMT response scales as a power law over a wide range of operating voltages. First, the mean areas of cosmic muon pulses are measured at 5-6 operating voltages, which are low enough to ensure that the PMT signals do not saturate. A power law function is then fit to these points, and extrapolated to the nominal operating voltage. Finally, this number is scaled by the per-channel SPE measurement to arrive at an estimate of the number of photoelectrons generated by a cosmic muon. The validity of the power law assumption is confirmed by separately fitting a power law function to the mean areas of SPE pulses over a range of voltages near the nominal operating voltage. The fitted exponent is found to be consistent with that from the fit to the cosmic muon pulse areas for all bars. The calibrated value of $\left\langle\mathrm{N}_{\mathrm{PE}}\right\rangle$ for a beam muon traversing the full $80 \mathrm{~cm}$ length of a bar varies from 22000 to 82000 ; this means that $\left\langle\mathrm{N}_{\mathrm{PE}}\right\rangle=1$ is expected in the bars for particles of charge $Q / e \sim 0.004-0.007$.

The dominant source of uncertainty in each bar's $\left\langle\mathrm{N}_{\mathrm{PE}}\right\rangle$ measurement is the statistical uncertainty from the power law fit and extrapolation, which is $10 \%-20 \%$ depending on the channel, and is uncorrelated between channels. Smaller uncertainties, generally on the order of a few percent, come from differences in the residual magnetic field between calibration runs and data-taking runs; the effect of a low-pass filter applied to the waveforms; and differences between the in situ and lab-based SPE measurements. These are correlated between bars with PMTs of the same type. Time dependent drifts in the response, inferred from variations in the dark count rate, are subdominant.

The timing of the PMTs must also be calibrated. The calibration procedure is designed such that a particle traveling near the speed of light through the detector from the CMS IP should have the same time value in every bar, panel and slab. This calibration is performed using both beam and cosmic muons. Figure 4 shows the time difference between a muon pulse in layer 3 compared to a muon pulse in layer 1, where the events have been categorized as either beam or cosmic muons based on the timing of the pulses in the slabs. The resolution in the time difference between layers is approximately 4 ns for beam muons which travel through the detector from the CMS IP. An additional correction is applied to account for the dependence of the timing of the pulses on their size. This correction is derived using secondary particles that result from the interactions of beam muons with the detector as they traverse it. The timing resolution degrades as the size of the pulses gets smaller; the resolution of the lowest $n_{\text {pe }}$ pulses passing the selection outlined in Sec. $\mathrm{V}$ is $\sim 15 \mathrm{~ns}$. The modeling of the timing of these secondaries is used to derive a systematic uncertainty in the timing in simulation.

The trigger efficiency is measured as a function of $n_{\mathrm{pe}}$ for each channel by comparing the rate between dedicated triple-coincidence runs and double-coincidence runs. For the triple-coincidence runs, the trigger decision is based on coincident signals in two "tag-channels" and one "probe channel," whose trigger efficiency is to be measured. For the double coincidence runs, the trigger decision involves only the two tag channels. The trigger efficiency for each channel is given by the ratio of the pulse rate when the 


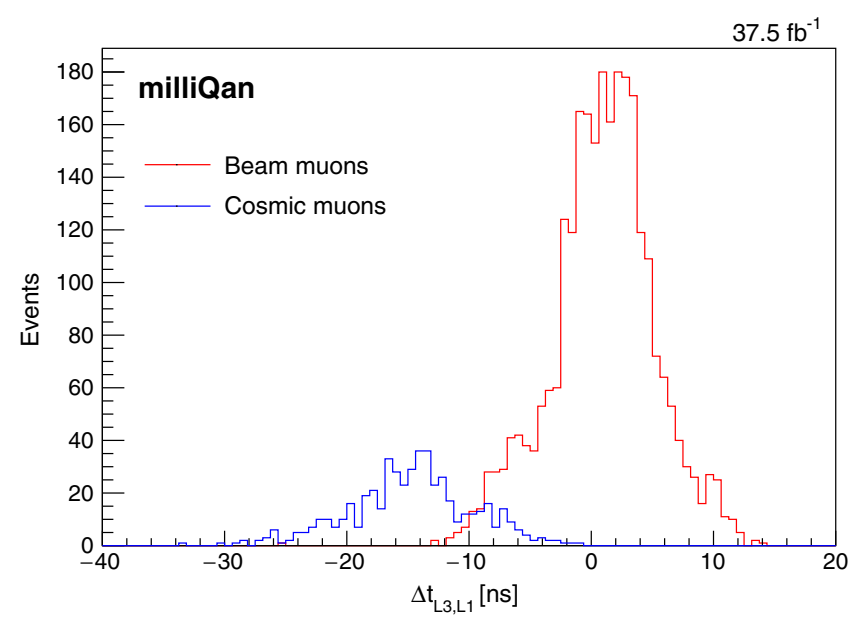

FIG. 4. Time difference, measured in a combination of the beam-on and beam-off datasets, between layer 3 and layer 1 for beam (red) and cosmic (blue) muons that travel through the detector.

probe channel is required in the trigger over the pulse rate when the probe channel is not required in the trigger. This efficiency is used to reweight the simulated samples of signals from millicharged particles described in Sec. IV B. The trigger efficiency is measured to reach $100 \%$ for $n_{\mathrm{pe}} \gtrsim 2$ for all channels.

\section{SIMULATION}

\section{A. Event generation}

We generate pair production of millicharged particles in $13 \mathrm{TeV}$ pp collisions through the Drell-Yan process, as well as from $\Upsilon, \mathrm{J} / \psi, \psi(2 \mathrm{~S}), \phi, \rho$, and $\omega$ decays into $\chi^{+} \chi^{-}$, and from Dalitz decays of $\pi^{0}, \eta, \eta^{\prime}$, and $\omega$.

Drell-Yan events are generated assuming that $\chi \mathrm{s}$ are isospin singlet fermions, using the Lagrangian of Ref. [37] implemented in the MADGRAPH5_aMC@NLO [38] event generator, with a minimum invariant mass requirement on the millicharged pair of $2 \mathrm{GeV} / c^{2}$. Because of the limited integrated luminosity and the small size of the demonstrator, there is essentially no sensitivity to Drell-Yan production of millicharged particles in the dataset discussed in this paper.

The production cross section and transverse momentum $p_{\mathrm{T}}$ distribution of $\mathrm{J} / \psi$ and $\psi(2 \mathrm{~S})$ in the central rapidity $y$ region is taken from calculations of charmonium production from direct processes [39-41] and from bottom hadron decays [42-44], including theoretical uncertainties. Theoretical calculations of bottomonium production [45] are not reliable at low transverse momentum $\left(p_{\mathrm{T}}<15 \mathrm{GeV}\right)$ [46], where most of the cross section lies. As a result, for $p_{\mathrm{T}}>20 \mathrm{GeV}$ we use the cross sections and $p_{\mathrm{T}}$ spectra measured at a center-of-mass energy of $13 \mathrm{TeV}$ [47]; at lower $p_{\mathrm{T}}$ we use measurements from $7 \mathrm{TeV}$ data
$[48,49]$, rescaled using the measured ratio of 13 to $7 \mathrm{TeV}$ cross sections at slightly higher rapidity $(2<y<2.5)$ [50].

All relevant light flavor mesons except $\phi$ mesons are generated with the minimum bias PYTHIA8 generator [51] with the Monash 2013 tune [52]. This is the tune that gives the best agreement with several measurements of light meson rates and $p_{\mathrm{T}}$ spectra at the LHC, albeit in most cases at center-of-mass energies lower than $13 \mathrm{TeV}$ [53-56]. The Monte Carlo spectra for $\eta(\rho, \omega)$ with $p_{\mathrm{T}}<3$ (1) $\mathrm{GeV}$ are scaled down by factors as large as 2 , based on the experimental results cited above. On the other hand, the production of $\phi$ mesons is modeled with the minimum bias PYTHIA6 generator [57] with the DW tune [58], since this Monte Carlo setup best reproduces $\phi$ meson data [59]. All PYTHIA Monte Carlo generations are normalized to a minimum bias cross section of $80 \pm 10 \mathrm{mb}$ based on a measurement by ATLAS [60], with an uncertainty taken to cover the difference with respect to a similar CMS measurement [61]. We assess an additional 30\% uncertainty in the overall rate of each process to account for remaining differences between experimental measurements and PYTHIA predictions of the rates of light mesons per minimum bias event, based on the references cited above.

The branching fractions for all vector meson decays, $V \rightarrow \chi^{+} \chi^{-}$, as a function of the $\chi$ mass are calculated using the Van Royen-Weisskopf formula [62], normalized to the PDG value of the branching fraction for $V \rightarrow e^{+} e^{-}$[63], and rescaled appropriately for the assumed charge of the $\chi$. The branching fractions for meson Dalitz decays, e.g., $\eta^{\prime} \rightarrow$ $\chi^{+} \chi^{-} \gamma$ or $\omega \rightarrow \chi^{+} \chi^{-} \pi^{0}$, as well as the $\chi^{+} \chi^{-}$invariant mass distributions in these decays are modeled as a function of the $\chi$ mass and charge using the partial width for decays into photons, e.g., $\eta^{\prime} \rightarrow \gamma \gamma$ or $\omega \rightarrow \pi^{0} \gamma$ [64], assuming a vector dominance model for the form factors.

Production cross sections of millicharged particles from different processes are summarized in Fig. 5. The possible contribution from millicharged particle production in EM showers in the CMS calorimeters generated by particles from pp collisions is not considered in this analysis.

We also generate inclusive muon events $(\mathrm{pp} \rightarrow \mathrm{X} \rightarrow \mu$ ) that are used for calibration and background studies. The same theoretical calculation used to obtain the differential cross sections of $\mathrm{J} / \psi$ and $\psi(2 \mathrm{~S})$ from bottom hadron decays is also used to generate muons from bottom and charm hadron decays, while muons from decays in flight of pions and kaons are generated with PYTHIA8 using the Monash 2013 tune. Muons from $\mathrm{W}$ and $\mathrm{Z}$ decays are taken from MADGRAPH5, though these electroweak processes contribute only $\sim 3 \%$ of the total muon flux. Finally, muons are generated using an appropriate angular distribution to simulate cosmic ray events. This angular distribution is derived by assuming a $\cos ^{2}\left(\theta_{\text {zenith }}\right)$ distribution at the surface and propagating the muons to the demonstrator using the method described in Sec. IV B. 


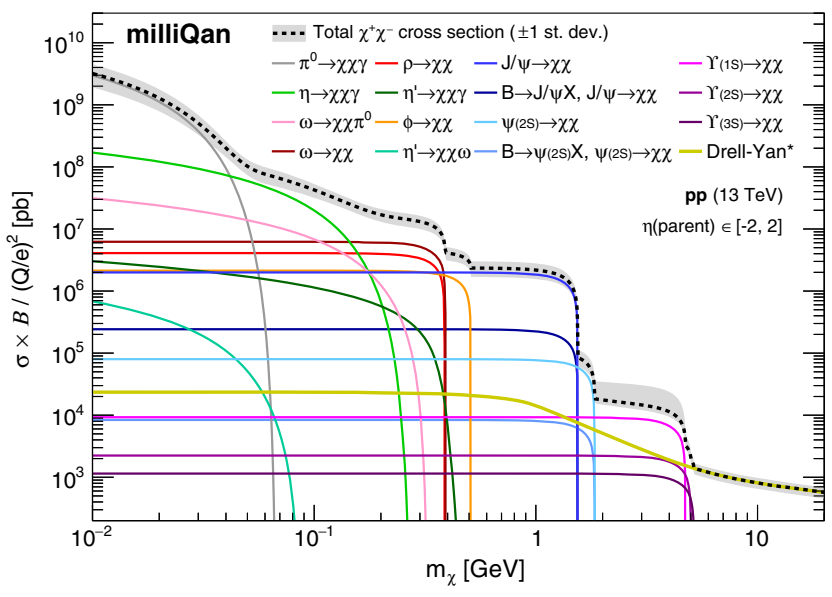

FIG. 5. Cross section times branching ratios for production of different particles with $|\eta|<2$ decaying into $\chi^{+} \chi^{-}$pairs, as a function of $\chi$ mass. For Drell-Yan the plotted cross section requires at least one of the $\chi$ s to have $|\eta|<1$.

\section{B. Detector response}

Generated particles are propagated through a simplified model of the material in the CMS detector, including the magnetic field, and the $17 \mathrm{~m}$ of rock between the CMS cavern and the demonstrator.

Propagation is performed with fourth-order Runge-Kutta integration, incorporating the effects of the magnetic field, multiple scattering, and energy loss. Particles are propagated until $2 \mathrm{~m}$ before the face of the demonstrator, after which they are fed into a full GEANT4 [65] simulation of the remaining rock, the drainage gallery where the demonstrator is located, and the demonstrator itself. Similarly, muons from cosmic ray showers are propagated from the surface of the earth to a plane $1 \mathrm{~m}$ above the top of the cavern. Muon interactions in the cavern walls generate showers of gamma rays and electrons that significantly contribute to the cosmic ray background. A simulated cosmic ray shower event is shown in Fig. 6.

The simulation parameters are nominally configured to be consistent with EJ-200 scintillator and existing measurements for Tyvek reflectivity and each PMT species' quantum efficiency [66]. Overall photon propagation is handled using GEANT4's UNIFIED optical model [67]. Photon propagation is further calibrated by measuring the effects of scintillator roughness and wrapping quality on light attenuation in the scintillator bars and matching the effect in simulation. These measurements indicated a wrapping reflectivity $R=0.97$ and scintillator roughness $\sigma=1 \%$, typical values for Tyvek and EJ-200 [68]. A perchannel calibration is applied to each PMT quantum efficiency so that the measured $\left\langle\mathrm{N}_{\mathrm{PE}}\right\rangle$ values agree between data and simulation.

The electronic response is simulated using SPE waveform templates measured on a test bench with an LED. SPE templates for each GEANT4 photoelectron are added

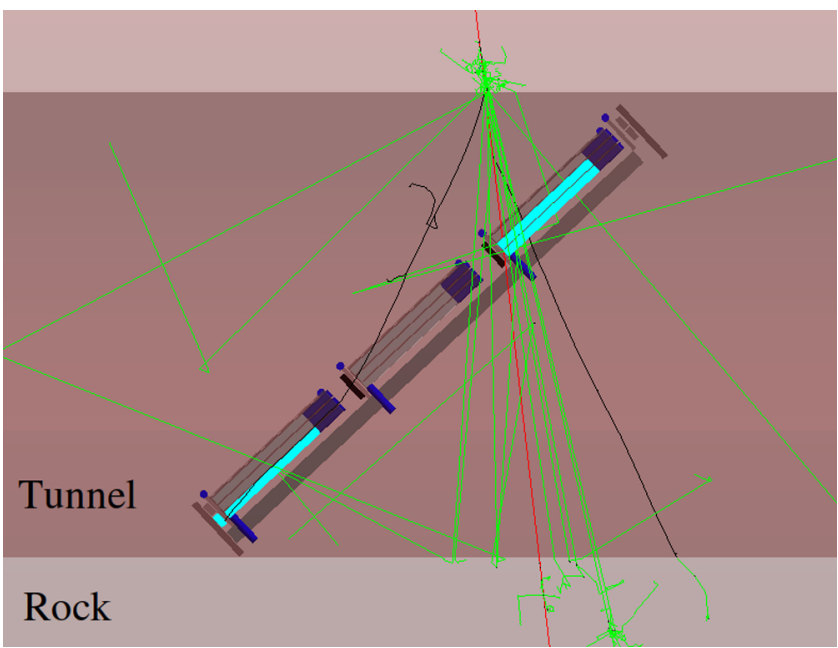

FIG. 6. Simulation of the milliQan demonstrator response to a simulated cosmic ray shower event. The incident muon (red) interacts with the cavern walls to produce a shower. Electrons (black) and gamma rays (green) generated in the rock are a significant background source. The interactions of muons and shower particles with detector material produce scintillation photons (cyan).

together, with appropriate arrival times, and including the individual PMT calibration described in Sec. III. The resulting waveform is then added to a randomly selected zero-bias data waveform to properly account for electronic noise.

\section{Validation}

The simulation is validated by studying beam muons as well as cosmic ray events. The absolute rate of beam muons passing through all four slabs is compared with the rate predicted from a simulated sample of muon production from heavy-flavor and electroweak decays, as well as meson decays in flight, as described in Sec. IV B. We measure a rate of $0.20 \pm 0.01$ muons $/ \mathrm{pb}^{-1}$, based on a sample of 7363 muons, in agreement with the prediction of $0.25 \pm 0.08$ muons $/ \mathrm{pb}^{-1}$. The dominant uncertainties in this prediction arise from the uncertainty in the $b \bar{b}$ cross section (21\%) and from the modeling of the material between the CMS IP and milliQan (25\%). This last uncertainty is derived from a $7 \%$ variation in the total amount of intervening material, which is in turn due primarily to uncertainties in both the thickness and density of the rock layer. This $7 \%$ variation corresponds to a $25 \%$ uncertainty in the muon rate because of the steeply falling muon momentum distribution. This same variation is used to derive a systematic uncertainty in the predicted signal yields, though in that case the effect is much smaller because of the smaller charge of the $\chi$.

We additionally perform a comparison of the angular distribution of beam muon trajectories, in order to probe the scattering and magnetic field modeling in simulation and 


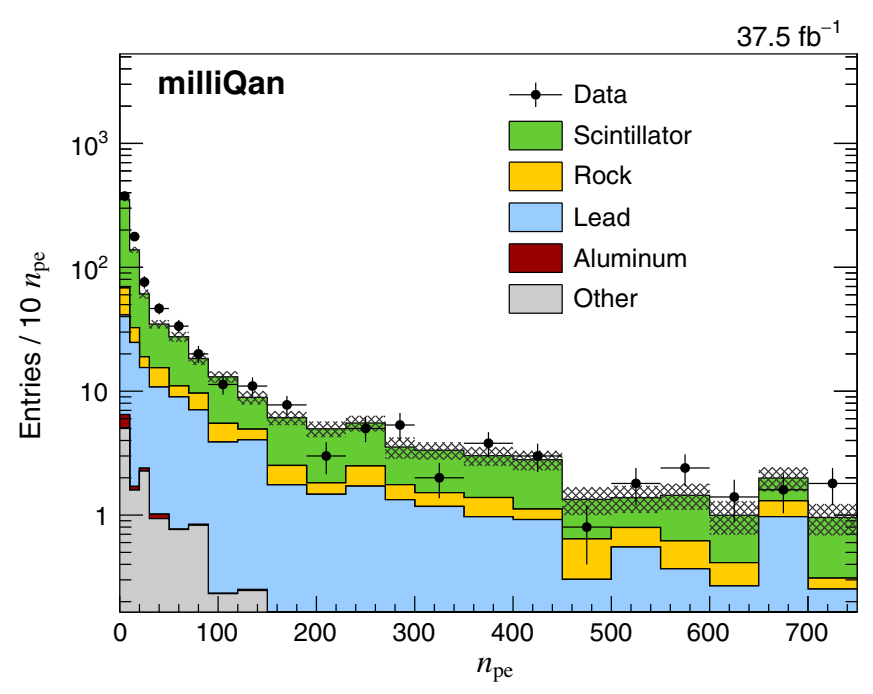

FIG. 7. Comparison of data and simulation $n_{\mathrm{pe}}$ distributions in events with a tagged throughgoing beam muon, for bars that do not contain a pulse consistent with originating from a muon, and are not neighboring any such bars. Note that only 783 out of 7363 tagged muons produced detectable showers entering this figure. Simulation events are categorized based on the material in which the particle(s) that produced the pulse originated.

validate the alignment of the detector. We compare rates of muons passing through various subsets of bars that trace a range of angles through the detector. The rates of these paths in both the horizontal and vertical directions are measured to be consistent within statistical uncertainties between data and simulation.

Finally, the GEANT4 modeling is validated by comparing distributions of photoelectron counts in bars near either beam or cosmic muon trajectories. Hits are expected from electrons and gamma rays produced as the muon travels through the rock or nearby detector material. An example comparison is shown in Fig. 7. Here we show $n_{\mathrm{pe}}$ distributions in data and simulation in events with a tagged beam muon, for bars that do not contain a pulse consistent with originating from a muon $\left(n_{\mathrm{pe}}<750\right)$, and are not neighboring any such bars. Contributions come primarily from electrons and gamma rays produced in scintillator material, rock, or the lead bricks. Good agreement is seen across a wide range of $n_{\text {pe }}$ levels.

\section{SEARCH FOR MILLICHARGED PARTICLES}

The search for millicharged particles looks for a signal with the signature of a pulse in each of the three layers of the demonstrator. A number of sources can produce such a signature:

(i) Each PMT has a dark current arising from effects such as the thermal emission of electrons from the cathode. The simplest background source comes from random overlap of three such dark rate pulses.
In addition, dark rate counts may overlap with two correlated pulses from another source.

(ii) Cosmic muon showers may generate a large number of gamma rays and electrons from an interaction of one or more cosmic ray muons with the rock in the milliQan cavern. This may cause a pulse in each layer of the demonstrator. Such a background could also be expected from a beam muon that travels close to the demonstrator.

(iii) Radiation in the cavern, scintillator bars or surrounding material can cause correlated deposits in several bars. The lead blocks placed between layers should reduce the probability of deposit in multiple layers arising from photons or electrons while the slabs provide shielding of neutrons.

(iv) Afterpulses, which are small pulses caused by positive ions generated by the ionization of residual gases in the PMT, can appear several hundred nanoseconds to over a few microseconds after the initial pulse. The afterpulses from correlated deposits in several PMTs may overlap and produce a signal-like signature in the demonstrator. For this to occur, the interaction event that gives rise to the afterpulses must not be triggered because, in this case, the afterpulses will fall in the $125 \mu$ s readout deadtime that follows each triggered event, and will not be recorded.

Selections are applied in order to reject contributions from these background sources. If there is a pulse within the acquisition time window in any panel, or in more than one bar in each layer, the event is rejected. These requirements reject backgrounds due to cosmic muon showers, which are expected to cause deposits across the detector. In addition, if there is a pulse in any slab within the acquisition time window consistent with originating from a muon $\left(n_{\mathrm{pe}}>250\right)$ the event is vetoed. This requirement rejects deposits due to beam and cosmic muons passing close to the bars. The bars with reconstructed pulses are required to be pointing to the CMS IP. This reduces the backgrounds from neutrons, cosmic muon showers and random overlap, while being efficient for signal, which typically has a small angular spread. To mitigate backgrounds from afterpulses, in each channel a requirement is made to reject the event if a pulse occurs before the window in which the pulse may be involved in the trigger decision. In addition, the first pulse in each channel must have the largest $n_{\text {pe }}$ value. Events that contain initial pulses in the bars with a large spread in $n_{\text {pe }}$ (maximum $n_{\mathrm{pe}} /$ minimum $n_{\mathrm{pe}}>10$ ) are vetoed to reject events containing contributions from different sources, such as dark rate overlap with shower deposits or deposits from two or more shower particles traveling through the demonstrator. Finally, the maximum calibrated time difference between the first bar pulse in each layer $\left(\Delta t_{\max }\right)$ is required to be less than $15 \mathrm{~ns}$, which is efficient for signals traveling through the detector from the CMS IP and forms a 
TABLE I. Sequential impact of each requirement on the number of events passing the selection criteria.

\begin{tabular}{|c|c|c|c|c|c|c|}
\hline & Selection & $\begin{array}{c}\text { Data } \\
\text { beam-on } \\
t=1106 \mathrm{~h}\end{array}$ & $\begin{array}{c}\text { Data } \\
\text { beam-off } \\
t=1042 \mathrm{~h}\end{array}$ & $\begin{array}{c}\text { Signal } \\
m_{\chi}=0.05 \mathrm{GeV} \\
Q / e=0.007\end{array}$ & $\begin{array}{c}\text { Signal } \\
m_{\chi}=1.0 \mathrm{GeV} \\
Q / e=0.02\end{array}$ & $\begin{array}{c}\text { Signal } \\
m_{\chi}=3.0 \mathrm{GeV} \\
Q / e=0.1\end{array}$ \\
\hline Common & $\geq 1$ hit per layer & 2003170 & 1939900 & 136.4 & 34.2 & 5.7 \\
\hline \multirow[t]{10}{*}{ Selections } & Exactly one hit per layer & 714991 & 698349 & 123.1 & 31.0 & 5.0 \\
\hline & Panel veto & 647936 & 632494 & 122.5 & 30.8 & 4.9 \\
\hline & First pulse is max & 418711 & 409296 & 114.3 & 30.6 & 4.8 \\
\hline & Veto early pulses & 301979 & 295040 & 113.9 & 30.6 & 4.8 \\
\hline & $\max n_{\mathrm{pe}} / \min n_{\mathrm{pe}}<10$ & 154203 & 150949 & 104.2 & 29.6 & 4.7 \\
\hline & $\Delta t_{\max }<15 \mathrm{~ns}$ & 5284 & 5161 & 72.8 & 28.4 & 4.4 \\
\hline & Slab muon veto & 5224 & 5153 & 72.8 & 28.4 & 4.4 \\
\hline & Straight path & 350 & 361 & 68.4 & 28.1 & 4.2 \\
\hline & $\mathrm{N}_{\mathrm{slab}}=0$ & 332 & 339 & 64.8 & 16.9 & 0.0 \\
\hline & $\mathrm{N}_{\text {slab }} \geq 1$ & 18 & 22 & 3.6 & 11.2 & 4.2 \\
\hline SR 1 & $\begin{array}{l}\mathrm{N}_{\text {slab }}=0 \text { and } \\
\quad \min n_{\mathrm{pe}} \in[2,20]\end{array}$ & 129 & 131 & 47.4 & 0.4 & 0.0 \\
\hline SR 2 & $\begin{array}{l}\mathrm{N}_{\text {slab }}=0 \text { and } \\
\quad \min n_{\mathrm{pe}}>20\end{array}$ & 52 & 45 & 0.0 & 16.5 & 0.0 \\
\hline SR 3 & $\begin{array}{l}\mathrm{N}_{\text {slab }}=1 \text { and } \\
\quad \min n_{\mathrm{pe}} \in[5,30]\end{array}$ & 8 & 9 & 1.1 & 0.5 & 0.0 \\
\hline SR 4 & $\begin{array}{l}\mathrm{N}_{\mathrm{slab}}=1 \text { and } \\
\quad \min n_{\mathrm{pe}}>30\end{array}$ & 4 & 4 & 0.0 & 8.7 & 0.0 \\
\hline SR 5 & $\mathrm{~N}_{\text {slab }} \geq 2$ & 1 & 1 & 0.0 & 2.0 & 4.2 \\
\hline
\end{tabular}

powerful rejection of backgrounds that have different paths through the detector, such as cosmic muon showers, or that have deposits in each layer that are uncorrelated in their timing, such as dark rate overlap.

Selected events are subsequently categorized into five signal regions (SRs) through requirements on both the number of slabs that contain a pulse and the minimum $n_{\mathrm{pe}}$ of the pulses in the three bars. This categorization allows for sensitivity to a wide range of charge values in the signal parameter space. The definitions of the five SRs are summarized in Table I. For events with a pulse in each of the three layers the selection criteria provide high efficiency for the targeted models while rejecting the background by more than 5 orders of magnitude.

Residual background passing selection is estimated for each signal region by measuring the pass/fail ratio of the timing requirement in events with a hit in each layer, consistent with signal requirements except that the bars do not form a pointing path towards the CMS IP, and then multiplying it by the number of events failing the timing selection that form a pointing path towards the CMS IP. This prediction method relies on the independence of the dominant backgrounds on the pointing path requirement. This method is used rather than taking the prediction from the beam-off dataset as it is robust against time dependent drifts in the background rate. However, the beam-off dataset provides a statistically independent sample to validate the prediction without contamination from signal. The results of the beam-off prediction for the SR are summarized in Table II. The uncertainty in the prediction reflects the limited statistics in the regions used to make the prediction. The prediction is shown to be in agreement with the observation for all validation regions. The level of agreement between prediction and observation in each validation region is used to derive a systematic uncertainty in the prediction.

Given the validation of the background prediction method with the beam-off dataset, the SR prediction is made using the beam-on dataset. The background contribution from beam processes is estimated from simulation to be less than $2 \%$ for all regions. Results are given in Table III. The predictions are seen to be consistent with those from the beam-off dataset (taking the 6\% difference in collection time into account), which provides additional confidence that the beam-based backgrounds are negligible. The uncertainty in the prediction reflects both the limited statistical power of the regions used for the

TABLE II. Summary of the results of the validation using the beam-off dataset. The systematic values are derived from the level of agreement between the prediction and observation.

\begin{tabular}{lccccc}
\hline \hline Region & $\mathrm{N}_{\text {slab }}$ & $\min n_{\mathrm{pe}}$ & Prediction & Observation & Systematic \\
\hline 1 & 0 & {$[2,20]$} & $121.2_{-5.9}^{+6.0}$ & 131 & $8 \%$ \\
2 & 0 & $>20$ & $47.4_{-4.8}^{+5.2}$ & 45 & $5 \%$ \\
3 & 1 & {$[5,30]$} & $7.8_{-1.8}^{+2.5}$ & 9 & $15 \%$ \\
4 & 1 & $>30$ & $2.7_{-1.1}^{+2.1}$ & 4 & $48 \%$ \\
5 & $\geq 2$ & $\cdots$ & $0.8_{-0.4}^{+1.4}$ & 1 & $25 \%$ \\
\hline \hline
\end{tabular}


TABLE III. Summary of the results of the signal region prediction.

\begin{tabular}{lcccc}
\hline \hline Region & $\mathrm{N}_{\text {slab }}$ & $\min n_{\mathrm{pe}}$ & Prediction & Observation \\
\hline 1 & 0 & {$[2,20]$} & $124_{-11}^{+11}$ & 129 \\
2 & 0 & $>20$ & $49.9_{-5.4}^{+6.0}$ & 52 \\
3 & 1 & {$[5,30]$} & $10.7_{-2.6}^{+3.6}$ & 8 \\
4 & 1 & $>30$ & $2.4_{-1.1}^{+2.1}$ & 4 \\
5 & $\geq 2$ & $\cdots$ & $0.0_{-0.0}^{+0.9}$ & 1 \\
\hline \hline
\end{tabular}

prediction as well as the systematic uncertainty derived from the validation using the beam-off dataset. The predictions are found to be consistent with the observations in all SRs.

\section{INTERPRETATION}

The search is interpreted using the signal model described in Sec. IV. The full set of selection criteria described in Sec. V is applied to each event. The efficiency to pass these criteria for three benchmark $\chi$ masses and charges is shown in Table I.

There are several sources of systematic uncertainty in the number of signal events entering the SRs. These are evaluated independently for each model point and are summarized below:

(i) Signal cross section (described in Sec. IVA): typically 15\%-30\% depending on the mass of the $\chi$.

(ii) Material interactions (described in Sec. IV C): typically $1 \%-5 \%$ depending on the charge and mass of the $\chi$.

(iii) Pulse timing (described in Sec. III): typically $1 \%-40 \%$ depending on the charge of the $\chi$.

(iv) $\left\langle\mathrm{N}_{\mathrm{PE}}\right\rangle$ calibration (described in Sec. III): $1 \%-50 \%$ depending on the SR populated by the $\chi$.

(v) Limited simulated sample size: up to $30 \%$.

Under the signal plus background hypothesis, a modified frequentist approach is used to determine observed upper limits at $95 \%$ confidence level on the cross section $(\sigma)$ to produce a pair of $\chi \mathrm{s}$, as a function of mass and charge. The approach uses the LHC-style profile likelihood ratio as the test statistic [69] and the criterion of the C.L. [70,71]. The observed upper limits are evaluated through the use of asymptotic formulas [72]. Figure 8 shows the exclusion at 95\% confidence level in mass and charge of the $\chi$. The exclusion is compared to existing constraints, showing new sensitivity for $\chi$ masses above $700 \mathrm{MeV}$.

\section{FUTURE PLANS}

In Refs. [30,37] we assumed the largest irreducible background to the signal would come from dark-current pulses in the PMTs. From experience gained by operating the demonstrator, we now know that an equally important background comes from correlated effects caused by

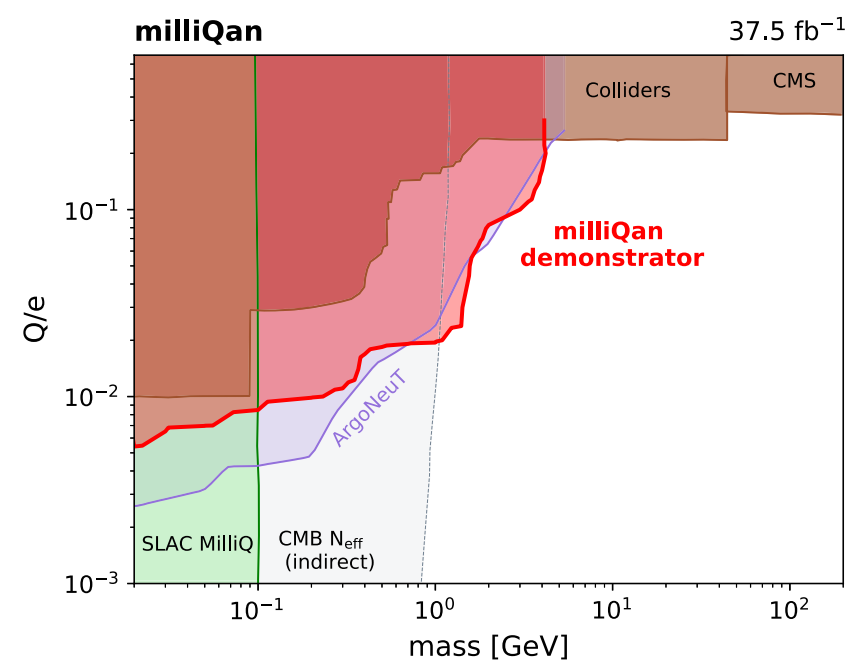

FIG. 8. Exclusion at 95\% confidence level compared to existing constraints from colliders, CMS, ArgoNeuT and SLAC MilliQ $[10-16,27]$ as well as the indirect constraint from the CMB relativistic degrees of freedom [26].

activity in the scintillator (from effects such as environmental radiation or cosmic muon showers). This realization prompted us to revisit the milliQan design, adding a fourth layer in order to mitigate the contribution from these correlated backgrounds.

We have studied the effect of adding a fourth layer with the demonstrator. The demonstrator has three rather than four layers so backgrounds are determined for threefold coincidence and then extended to fourfold using an additional pulse in a slab. The results of this study indicate that the contribution from pure dark-current overlap drops to a negligible level for the case of fourfold coincidence, even with the somewhat high-noise PMTs that are used in the demonstrator. The calculations presented in Refs. [30,37] remain a conservative estimate of the milliQan discovery potential since the background with four layers as measured with the demonstrator is significantly smaller than the estimate used in those simulations.

Given the experience obtained from the demonstrator, we are confident that the proposed full-scale detector will perform as expected provided sufficient funding becomes available.

\section{CONCLUSIONS}

We have deployed a prototype dedicated detector at the LHC to conduct the first search for elementary particles with charges much smaller than the electron charge at a hadron collider. We analyzed a data sample of protonproton collisions collected at $\sqrt{s}=13 \mathrm{TeV}$ provided by the LHC, corresponding to an integrated luminosity of $37.5 \mathrm{fb}^{-1}$. The existence of new particles with masses between 20 and $4700 \mathrm{MeV}$ is excluded at $95 \%$ confidence level for charges varying between $0.006 e$ and $0.3 e$, 
depending on mass. New sensitivity is achieved for masses larger than $700 \mathrm{MeV}$. The successful operation of the milliQan demonstrator and search carried out have shown the feasibility of a dedicated detector for millicharged particles at the LHC and provided important lessons for the design of the full detector.

\section{ACKNOWLEDGMENTS}

We congratulate our colleagues in the CERN accelerator departments for the excellent performance of the LHC and thank the technical and administrative staffs at CERN.
In addition, we gratefully acknowledge the CMS collaboration for supporting this endeavor by providing invaluable logistical and technical assistance. We also thank Eder Izaguirre and Itay Yavin for their enduring contributions to this idea. Finally, we acknowledge the following funding agencies who support the investigators that carried out this research in various capacities: FWO (Belgium) under the Excellence of Science EOS be.h Project No. 30820817; DFG and HGF (Germany); Swiss Funding Agencies (Switzerland); STFC (United Kingdom); DOE and NSF (USA); Lebanese University (Lebanon).
[1] N. Arkani-Hamed, D. P. Finkbeiner, T. R. Slatyer, and N. Weiner, A theory of dark matter, Phys. Rev. D 79, 015014 (2009).

[2] M. Pospelov and A. Ritz, Astrophysical signatures of secluded dark matter, Phys. Lett. B 671, 391 (2009).

[3] M. Battaglieri, A. Belloni, A. Chou et al., U.S. cosmic visions: New ideas in dark matter 2017: Community report, in U.S. Cosmic Visions: New Ideas in Dark Matter (Fermilab, Batavia, 2017), Vol. 7.

[4] J. Beacham, C. Burrage, D. Curtin et al., Physics beyond colliders at CERN: Beyond the standard model working group report, J. Phys. G 47, 010501 (2020).

[5] European Strategy for Particle Physics Preparatory Group, Physics Briefing Book (CERN, Geneva, 2019).

[6] L. B. Okun, Limits of electrodynamics: Paraphotons?, Zh. Eksp. Teor. Fiz. 83, 892 (1982) [Sov. Phys. JETP 56, 502 (1982)], https://inspirehep.net/literature/177918.

[7] E. Izaguirre and I. Yavin, New window to millicharged particles at the LHC, Phys. Rev. D 92, 035014 (2015).

[8] B. Holdom, Two U(1)'s and epsilon charge shifts, Phys. Lett. B 166, 196 (1986).

[9] S. Davidson and M. E. Peskin, Astrophysical bounds on millicharged particles in models with a paraphoton, Phys. Rev. D 49, 2114 (1994).

[10] A. Prinz, R. Baggs, J. Ballam et al., Search for Millicharged Particles at SLAC, Phys. Rev. Lett. 81, 1175 (1998).

[11] R. Essig, J. A. Jaros, W. Wester et al., Working group report: New light weakly coupled particles, in Community Summer Study 2013: Snowmass on the Mississippi (Fermilab, Batavia, 2013), Vol. 10.

[12] S. Chatrchyan (CMS Collaboration), Search for fractionally charged particles in pp collisions at $\sqrt{s}=7 \mathrm{TeV}$, Phys. Rev. D 87, 092008 (2013).

[13] S. Chatrchyan (CMS Collaboration), Searches for longlived charged particles in pp collisions at $\sqrt{s}=7$ and $8 \mathrm{TeV}$, J. High Energy Phys. 07 (2013) 122.

[14] R. Acciarri, C. Adams, J. Asaadi et al., Improved Limits on Millicharged Particles Using the ArgoNeuT Experiment at Fermilab, Phys. Rev. Lett. 124, 131801 (2020).

[15] S. Davidson, S. Hannestad, and G. Raffelt, Updated bounds on millicharged particles, J. High Energy Phys. 05 (2000) 003.
[16] A. Badertscher, P. Crivelli, W. Fetscher, U. Gendotti, S. N. Gninenko, V. Postoev, A. Rubbia, V. Samoylenko, and D. Sillou, An improved limit on invisible decays of positronium, Phys. Rev. D 75, 032004 (2007).

[17] G. Magill, R. Plestid, M. Pospelov, and Y.-D. Tsai, Millicharged Particles in Neutrino Experiments, Phys. Rev. Lett. 122, 071801 (2019).

[18] S. Davidson, B. Campbell, and D. C. Bailey, Limits on particles of small electric charge, Phys. Rev. D 43, 2314 (1991).

[19] R. Mohapatra and I. Rothstein, Astrophysical constraints on minicharged particles, Phys. Lett. B 247, 593 (1990).

[20] R. Agnese, A. Anderson, D. Balakishiyeva et al., First Direct Limits on Lightly Ionizing Particles with Electric Charge Less than e/6, Phys. Rev. Lett. 114, 111302 (2015).

[21] T. Emken, R. Essig, C. Kouvaris, and M. Sholapurkar, Direct detection of strongly interacting sub-gev dark matter via electron recoils, J. Cosmol. Astropart. Phys. 09 (2019) 070.

[22] L. Singh (TEXONO Collaboration), Constraints on millicharged particles with low-threshold germanium detectors at Kuo-Sheng Reactor Neutrino Laboratory, Phys. Rev. D 99, 032009 (2019).

[23] S. I. Alvis (Majorana Collaboration), First Limit on the Direct Detection of Lightly Ionizing Particles for Electric Charge as Low as $e / 1000$ with the Majorana Demonstrator, Phys. Rev. Lett. 120, 211804 (2018).

[24] P. C. Kim, E. R. Lee, I. T. Lee, M. L. Perl, V. Halyo, and D. Loomba, Search for Fractional-Charge Particles in Meteoritic Material, Phys. Rev. Lett. 99, 161804 (2007).

[25] D. C. Moore, A. D. Rider, and G. Gratta, Search for Millicharged Particles Using Optically Levitated Microspheres, Phys. Rev. Lett. 113, 251801 (2014).

[26] C. Brust, D. E. Kaplan, and M. T. Walters, New light species and the CMB, J. High Energy Phys. 12 (2013) 058.

[27] H. Vogel and J. Redondo, Dark radiation constraints on minicharged particles in models with a hidden photon, J. Cosmol. Astropart. Phys. 02 (2014) 029.

[28] G. Aad (ATLAS Collaboration), The ATLAS experiment at the CERN large hadron collider, J. Instrum. 3, S08003 (2008). 
[29] S. Chatrchyan (CMS Collaboration), The CMS experiment at the CERN LHC, J. Instrum. 3, S08004 (2008).

[30] A. Ball, J. Brooke, C. Campagnari et al., A letter of intent to install a millicharged particle detector at LHC P5, arXiv: 1607.04669.

[31] Eljen Technology, https://eljentechnology.com/products/ plastic-scintillators/ej-200-ej-204-ej-208-ej-212.

[32] Hamamatsu Photonics K. K., https://www.hamamatsu .com/.

[33] ET Enterprises, Ltd., http://et-enterprises.com/.

[34] CAEN S.p.A, https://www.caen.it/products/v1743/.

[35] CAEN S.p.A, https://www.caen.it/products/sy5527/.

[36] R. Saldanha, L. Grandi, Y. Guardincerri, and T. Wester, Model independent approach to the single photoelectron calibration of photomultiplier tubes, Nucl. Instrum. Methods Phys. Res., Sect. A 863, 35 (2017).

[37] A. Haas, C. S. Hill, E. Izaguirre, and I. Yavin, Looking for millicharged particles with a new experiment at the LHC, Phys. Lett. B 746, 117 (2015).

[38] J. Alwall, R. Frederix, S. Frixione, V. Hirschi, F. Maltoni, O. Mattelaer, H.-S. Shao, T. Stelzer, P. Torrielli, and M. Zaro, The automated computation of tree-level and next-toleading order differential cross sections, and their matching to parton shower simulations, J. High Energy Phys. 07 (2014) 079.

[39] Y.-Q. Ma, K. Wang, and K.-T. Chao, A complete NLO calculation of the $\mathrm{J} / \psi$ and $\psi^{\prime}$ production at hadron colliders, Phys. Rev. D 84, 114001 (2011).

[40] Y.-Q. Ma, K. Wang, and K.-T. Chao, J/ $\psi\left(\psi^{\prime}\right)$ Production at the Tevatron and LHC at $\mathcal{O}\left(\alpha_{s}^{4} v^{4}\right)$ in Nonrelativistic QCD, Phys. Rev. Lett. 106, 042002 (2011).

[41] Y.-Q. Ma and R. Venugopalan, Comprehensive Description of $\mathrm{J} / \psi$ Production in Proton-Proton Collisions at Collider Energies, Phys. Rev. Lett. 113, 192301 (2014).

[42] M. Cacciari, M. L. Mangano, and P. Nason, Gluon PDF constraints from the ratio of forward heavy-quark production at the LHC at $\sqrt{s}=7$ and $13 \mathrm{TeV}$, Eur. Phys. J. C 75, 610 (2015).

[43] M. Cacciari, S. Frixione, N. Houdeau, M. L. Mangano, P. Nason, and G. Ridolfi, Theoretical predictions for charm and bottom production at the LHC, J. High Energy Phys. 10 (2012) 137.

[44] M. Cacciari, S. Frixione, and P. Nason, The $p_{\mathrm{T}}$ spectrum in heavy flavor photoproduction, J. High Energy Phys. 03 (2001) 006.

[45] H. Han, Y.-Q. Ma, C. Meng, H.-S. Shao, Y.-J. Zhang, and K.-T. Chao, $\Upsilon(n S)$ and $\chi_{b}(n P)$ production at hadron colliders in nonrelativistic QCD, Phys. Rev. D 94, 014028 (2016).

[46] Y.-Q. Ma (private communication).

[47] S. Chatrchyan (CMS Collaboration), Measurement of quarkonium production cross sections in pp collisions at $\sqrt{s}=13 \mathrm{TeV}$, Phys. Lett. B 780, 251 (2018).

[48] G. Aad (ATLAS Collaboration), Measurement of the $\Upsilon(1 \mathrm{~S})$ production cross section in pp collisions at $\sqrt{s}=7 \mathrm{TeV}$ in ATLAS, Phys. Lett. B 705, 9 (2011).
[49] G Aad (ATLAS Collaboration), Measurement of Upsilon production in $7 \mathrm{TeV}$ pp collisions at ATLAS, Phys. Rev. D 87, 052004 (2013).

[50] R. Aaij (LHCb Collaboration), Measurement of $\Upsilon$ production in pp collisions at $\sqrt{s}=13 \mathrm{TeV}$, J. High Energy Phys. 07 (2018) 134; Erratum, J. High Energy Phys. 05 (2019) 076.

[51] T. Sjöstrand, S. Mrenna, and P. Skands, A brief introduction to PYTHia8.1, Comput. Phys. Commun. 178, 852 (2008).

[52] P. Skands, S. Carrazza, and J. Rojo, Tuning PYTHIA8.1: The Monash 2013 Tune, Eur. Phys. J. C 74, 3024 (2014).

[53] S. Acharya (ALICE Collaboration), $\pi^{0}$ and $\eta$ meson production in proton-proton collisions at $\sqrt{s}=8 \mathrm{TeV}$, Eur. Phys. J. C 78, 263 (2018).

[54] S. Acharya (ALICE Collaboration), Production of the $\rho(770)^{0}$ meson in $\mathrm{pp}$ and $\mathrm{Pb}-\mathrm{Pb}$ collisions at $\sqrt{s_{\mathrm{NN}}}=$ 2.76 TeV, Phys. Rev. C 99, 064901 (2019).

[55] S. Acharya (ALICE Collaboration), Production of $\omega(782)$ in pp collisions at $\sqrt{s}=7 \mathrm{TeV}$, CERN Technical Report No. ALICE-PUBLIC-2018-004, 2018.

[56] S. Chatrchyan (CMS Collaboration), Measurement of charged pion, kaon, and proton production in protonproton collisions at $\sqrt{s}=13 \mathrm{TeV}$, Phys. Rev. D 96, 112003 (2017).

[57] T. Sjöstrand, S. Mrenna, and P. Skands, PYTHIA6.4 physics and manual, J. High Energy Phys. 05 (2006) 026.

[58] M. G. Albrow, M. Begel, D. Bourilkov et al., Tevatronfor-LHC report of the QCD working group, arXiv:hep-ph/ 0610012.

[59] G. Aad (ATLAS Collaboration), The differential production cross section of the $\phi$ (1020) meson in $\sqrt{s}=7 \mathrm{TeV}$ pp collisions measured with the ATLAS detector, Eur. Phys. J. C 74, 2895 (2014).

[60] G. Aad (ATLAS Collaboration), Measurement of the Inelastic Proton-Proton Cross Section at $\sqrt{s}=13 \mathrm{TeV}$ with the ATLAS Detector at the LHC, Phys. Rev. Lett. 117, 182002 (2016).

[61] S. Chatrchyan (CMS Collaboration), Measurement of the inelastic proton-proton cross section at $\sqrt{s}=13 \mathrm{TeV}$, CERN Technical Report No. CMS-PAS-FSQ-15-005, 2016.

[62] R. Van Royen and V. F. Weisskopf, Hadron decay processes and the quark model, Nuovo Cimento A 50, 617 (1967); Erratum, Nuovo Cimento A 51, 583 (1967).

[63] M. Tanabashi et al. (Particle Data Group), Review of particle physics, Phys. Rev. D 98, 030001 (2018).

[64] L. G. Landsberg, Electromagnetic decays of light mesons, Phys. Rep. 128, 301 (1985).

[65] S. Agostinelli, J. Allison, K. Amako et al., GEANT4A simulation toolkit, Nucl. Instrum. Methods Phys. Res., Sect. A 506, 250 (2003).

[66] M. Janecek and W. W. Moses, Optical reflectance measurements for commonly used reflectors, IEEE Trans. Nucl. Sci. 55, 2432 (2008).

[67] A. Levin and C. Moisan, A more physical approach to model the surface treatment of scintillation counters and 
its implementation into DETECT, in 1996 IEEE Nuclear Science Symposium. Conference Record (IEEE, Anaheim, CA, 1996), Vol. 2, p. 702.

[68] P. Papacz, Optimization of the particle detection efficiency for scintillation detectors with SiPM readout, Master's thesis, RWTH Aachen University, 2010.

[69] G. Aad (ATLAS and CMS Collaborations), Procedure for the LHC Higgs boson search combination in Summer 2011, CERN Technical Reports No. CMS-NOTE-2011-005 and No. ATL-PHYS-PUB-2011-11, 2011.
[70] T. Junk, Confidence level computation for combining searches with small statistics, Nucl. Instrum. Methods Phys. Res., Sect. A 434, 435 (1999).

[71] A. L. Read, Presentation of search results: The CLs technique, J. Phys. G 28, 2693 (2002).

[72] G. Cowan, K. Cranmer, E. Gross, and O. Vitells, Asymptotic formulas for likelihood-based tests of new physics, Eur. Phys. J. C 71, 1554 (2011); Erratum, Eur. Phys. J. C 73, 2501 (2013). 